\title{
Estimation of hepatitis $\mathbf{E}$ virus transmission among pigs due to contact-exposure
}

\author{
Martijn BouwknegT ${ }^{1,2 *}$, Klaas Frankena ${ }^{2}$, Saskia A. RutJes ${ }^{1}$, \\ Gerard J. WellenberG ${ }^{3} \dagger$, Ana Maria De Roda Husman ${ }^{1}$, \\ Wim H.M. VAN DER POEL ${ }^{3}$, Mart C.M. DE JONG ${ }^{2,3}$
${ }^{1}$ Laboratory for Zoonoses and Environmental Microbiology, Centre for Infectious Disease Control Netherlands, National Institute for Public Health and the Environment, Bilthoven, The Netherlands
${ }^{2}$ Quantitative Veterinary Epidemiology Group, Wageningen Institute of Animal Sciences, Wageningen, The Netherlands
${ }^{3}$ Animal Sciences Group of Wageningen UR, Department of Infectious Diseases, Lelystad, The Netherlands

(Received 19 August 2007; accepted 19 March 2008)

\begin{abstract}
Locally acquired hepatitis E in humans from industrialized countries has been repeatedly suggested to originate from pigs. Pigs may serve as a reservoir of hepatitis E virus (HEV) for humans when a typical infected pig causes on average more than one newly infected pig, a property that is expressed by the basic reproduction ratio $R_{0}$. In this study, $R_{0}$ for HEV transmission among pigs was estimated from chains of one-to-one transmission experiments in two blocks of five chains each. Per chain, susceptible first-generation contact pigs were contact-exposed to intravenously inoculated pigs, subsequently susceptible second-generation contact pigs were contact-exposed to infected first-generation contact pigs, and lastly, susceptible third-generation contact pigs were contact-exposed to infected second-generation contact pigs. Thus, in the second and third link of the chain, HEV-transmission due to contact with a contact-infected pig was observed. Transmission of HEV was monitored by reverse transcriptase polymerase chain reaction (RT-PCR) on individual faecal samples taken every two/three days. For susceptible pigs, the average period between exposure to an infectious pig and HEV excretion was six days (standard deviation: 4). The length of HEV-excretion (i.e. infectious period) was estimated at 49 days (95\% confidence interval (CI): 17-141) for block 1 and 13 days (95\% CI: 11-17) for block 2 . The $R_{0}$ for contact-exposure was estimated to be 8.8 (95\% CI: 4-19), showing the potential of HEV to cause epidemics in populations of pigs.
\end{abstract}

hepatitis E virus / transmission / contact-exposure / reproduction ratio / pigs

\section{INTRODUCTION}

Hepatitis E virus (HEV) is a cause of hepatitis among humans predominantly in developing countries. However, more and more $\mathrm{HEV}$ infections are being identified in industrialized countries. Partly, these infections result from travel to $\mathrm{HEV}$-endemic areas; for the other part, the sources of HEV are mostly unknown [17, 42]. Several animal species have been suggested as possible sources, including cattle, rodents, dogs, wild boar, deer, horses and domestic pigs $[2,25,33,36,41]$. Amongst these species, domestic pigs carry HEV most ubiquitously, with prevalence estimates of more than $50 \%$ for both the pig- and farm-level [27,32,37,38].

Because HEV strains from domestic pigs and humans show extensive similarity, zoonotic transmission has been suggested $[15,25,29]$. This suggestion is supported by successful experimental infection of

* Corresponding author: martijn.bouwknegt@ rivm.nl

$\dagger$ Current affiliation: Animal Health Service (GD), Deventer, The Netherlands

Article available at http://www.vetres.org or http://dx.doi.org/10.1051/vetres:2008017 
pigs with human HEV and nonhuman primates with porcine HEV [26]. Furthermore, exposure to domestic pigs was identified by serological studies as a potential causal factor for human cases of hepatitis $E$ $[5,12,28]$.

If human cases of hepatitis $E$ are caused by exposure to pigs, then at least HEV should persist and be transmitted within populations of pigs. In other words, pigs should be a true animal reservoir for $\mathrm{HEV}$, defined as a population of animals that can maintain HEV infection without the need of other HEV sources. To assess whether pigs are a true animal reservoir, two aspects need to be studied. First, the infected pigs should be able to transmit HEV to other pigs. Second, the contact structure within farms should allow persistence of HEV. Although the high level of HEV infection in domestic pigs strongly suggests that pigs meet these two requirements, crucial evidence to support this suggestion is lacking. Evidence can be provided by quantifying the basic reproduction ratio $\left(R_{0}\right)$, which defines the average total number of new infections caused by one typical infectious animal during its entire infectious period in a completely susceptible population $[1,24]$. When $R_{0}$ is larger than 1 , HEV transmission can be maintained among pigs. Estimates and confidence intervals for $R_{0}$ can be obtained by transmission experiments [8].

Published animal infection experiments with HEV have mainly used intravenous inoculation as the route of infection, but intrahepatic inoculation is also described [16]. The intravenous route is more efficient in causing $\mathrm{HEV}$ infection in pigs and primates than the oral route $[20,30]$. This higher efficiency is needed when one needs to be certain that HEV, when present, causes infection. However, the natural route for HEV-transmission is most likely faecal-oral $[6,31]$. The natural route of transmission is required to study transmission dynamics and dynamics of HEV infection in individual animals. Furthermore, infection due to contact-exposure has been observed previously $[20,26]$. Therefore, studies on HEV dynamics in pigs are ideally done with contactinfected pigs, using exposure of susceptible pigs to infectious pigs.

The objective of the current study was to quantify the transmission of HEV among pigs that were infected by exposure to an infectious pig. Therefore, a transmission chain was designed: susceptible first-generation contact pigs were contact-exposed to intravenously inoculated pigs; susceptible secondgeneration contact pigs were contact-exposed to infected first-generation contact pigs; and susceptible third-generation contact pigs were contact-exposed to infected secondgeneration contact pigs. Data from the transmission chains were used to estimate the period between infection and HEV excretion, the number of days of HEV excretion (i.e. infectious period) and ultimately $R_{0}$.

\section{MATERIALS AND METHODS}

\subsection{Stable design}

Power-calculations demonstrated that ten replicates of a transmission chain were needed, each replicate requiring a separate stable to avoid HEV-transmission between replicates. The stables were subdivided in three adjacent compartments of about $3 \mathrm{~m}^{2}$ each, with a main passage in front for access and waste removal (Fig. 1). All inner walls in the stable were made of new multiplex wood of $145 \mathrm{~cm}$ in height. The walls that separated compartments contained plastic windows to enable visual contact between pigs in neighbouring compartments (required by the ethical committee). Within compartments, all joints between walls and the floor were sealed. The floors of the stables were solid and a thin layer of saw dust as bedding was supplied (required by the ethical committee).

Four male pigs of 3-4 weeks of age were allotted to one stable one week before inoculation. All pigs were of about the same age at the start of the experiment. Two pigs were placed in the compartment furthest from the main entrance of the stable (compartment 1); each of the other two pigs was placed in one of the two remaining compartments. Faeces of pigs tested negative for the presence of HEV RNA at 14 days and 7 days before inoculation. Furthermore, serum samples 


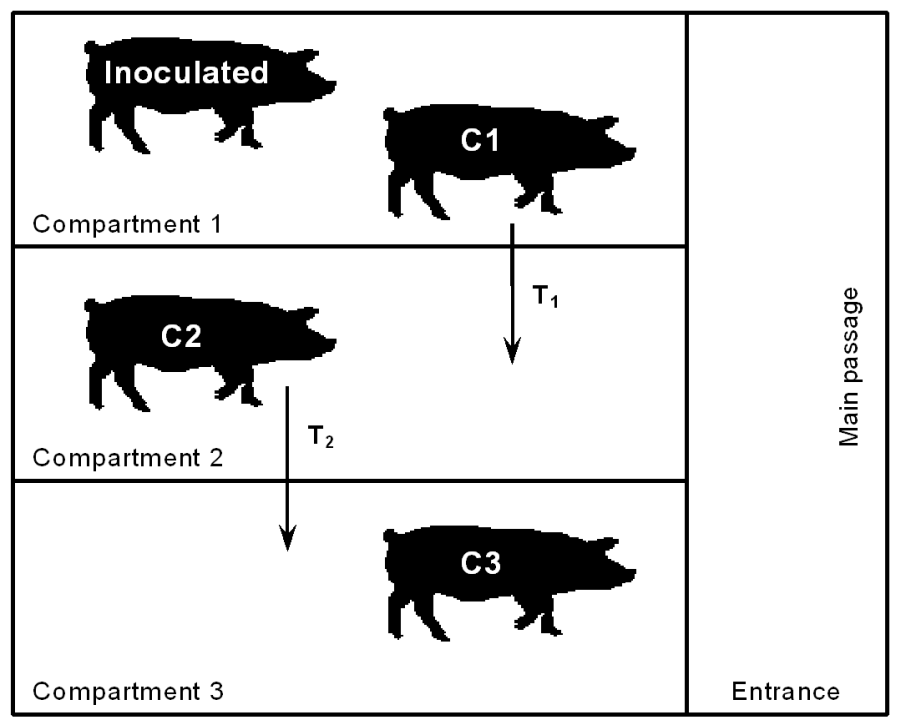

Figure 1. Schematic representation of the experimental setup to study transmission of hepatitis $\mathrm{E}$ virus in pigs. Intravenously inoculated pigs were to infect first-generation contact pigs (C1). Once infected, C1-pigs were transferred to the second compartment (at time $\mathrm{T}_{1}$ ) to infect second-generation contact pigs (C2). Once infected, C2-pigs were transferred to the third compartment (at time $\mathrm{T}_{2}$ ) to infect third-generation contact pigs (C3). Data on transmission from C1- to C2-pigs and from C2- to C3-pigs were used to estimate $R_{0}$ for hepatitis E virus.

collected at 7 or 4 days before inoculation tested negative for anti-HEV antibodies, indicating an absence of maternal immunity. Therefore, pigs were considered susceptible for HEV-infection.

Since only five stables were available simultaneously, the experiment was done in two separate blocks, using five stables per block. After the first block and before the second block, all stables were thoroughly cleaned with water containing hypochlorite, disinfected by fumigation with formaline, cleaned with water under high pressure and treated consecutively with acid, sodiumhydroxide and a detergent. Swab samples were taken from the walls, the floors, the drinking nipples, the boots, the disinfection tub, the ventilator and the manure pit, and were shown to be free of HEV RNA and therefore presumably HEV particles before the start of the second block.

This animal experiment was approved by the Ethics Committee on Animal Experiments of the Animal Sciences Group of Wageningen University and Research in Lelystad. The experiment was done in a BSL-2 facility.

\subsection{Virus}

HEV was isolated from a Dutch finishing pig and was characterized as genotype 3 (GenBank: DQ996399). To obtain sufficient virus for the experiment, the virus was amplified by intravenous inoculation in a caesarean-derived, colostrum deprived pig under high health conditions. The infected pig was euthanized 28 days post infection (dpi) and the liver was used to prepare inoculates. The pig tested negative for porcine reproductive and respiratory syndrome virus, porcine circovirus type 2 and enterovirus by reverse transcriptase polymerase chain reaction (RT-PCR).

To prepare the inoculates, the liver was grinded in EMEM (Gibco ${ }^{\circledR}$, Invitrogen ${ }^{\mathrm{TM}}$, Breda, The Netherlands) with a mortar and pestle. The suspension was transferred to a $15 \mathrm{~mL}$ tube. After 15 min of settlement, the supernatant was transferred to a new tube and filtered consecutively through microfilters of $0.45 \mu \mathrm{m}$ and $0.22 \mu \mathrm{m}$ pore size. The suspension was aliquoted in volumes of $2.5 \mathrm{~mL}$ and stored at $-70^{\circ} \mathrm{C}$. 
The inoculates contained about $10^{4}$ PCR detectable units of HEV RNA per $\mathrm{mL}$, quantified as the most probable number using maximum likelihood estimates based on the end-point dilution in RT-PCR [40]. Each intravenously inoculated pig received $2 \mathrm{~mL}$ of inoculum in the first block and $1 \mathrm{~mL}$ of inoculum in the second block, resulting in a $0.3 \log _{10}$ difference in HEV titre between the blocks. The inoculates were administered via the vena auricularis.

\subsection{Transmission chains}

In total, ten replicate transmission chains were done. Each transmission chain consisted of three one-to-one exposures, i.e. one susceptible pig was contact-exposed to one infectious pig. The infectious pigs for the first one-to-one exposure were created by intravenous inoculation of HEV (IV-pigs). After inoculation, the first-generation contact pigs $(\mathrm{C} 1)$ were contact-exposed to IV-pigs (Fig. 1). When C1-pigs excreted HEV RNA in faeces at three consecutive samplings, the pigs were assumed to be infected and infectious, and were transferred to the adjacent compartment with a second-generation contact pig (C2). When the C2-pigs excreted HEV RNA in faeces at three consecutive samplings, they were transferred to the adjacent compartment with a third-generation contact pig (C3). When a C3-pig excreted HEV RNA in faeces at three consecutive samplings, the transmission chain ended. Transmission chains also ended when an infectious pig stopped to excrete $\mathrm{HEV}$ in the faeces before infecting the contact pig. Pigs were euthanized after successful transmission of HEV to contact pigs, or when the transmission chain in that stable ended.

\subsection{Sample collection}

Individual faecal samples were collected from each pig in the transmission chain at seven days before infection and the day of infection. Upon inoculation on a Monday, animal-technicians collected individual faecal samples from the pigs in one-to-one experiments every Monday, Wednesday and Friday. When contact-exposed susceptible pigs were positive for HEV RNA in faeces at two consecutive samplings, a control sample was taken from the next-generation contact pig to examine the absence of HEV RNA.

Page 4 of 11 (page number not for citation purpose)
In each stable, samples were taken from the susceptible contact pig first, followed by the infectious pig. When a control sample from a nextgeneration contact pig was required, this sample was taken before the other samples. In each stable and at each sampling, new disposable overalls, gloves and facial masks were used. Furthermore, a pair of boots was present in each stable, which were cleaned and disinfected after each sampling. These measures should minimize the risk of transmission of HEV by the animal technicians.

Faecal samples were cooled on ice, transported to the laboratory, and processed immediately. Samples were mixed 1:1 in tryptone soya broth with $20 \%$ glycerol. About $200 \mathrm{mg}$ of this 1:1-solution was further diluted 1:5 to obtain a $10 \%$ suspension in Hank's balanced salt solution containing $50 \mu \mathrm{g} / \mathrm{mL}$ gentamycine; the remainder was stored at $-70{ }^{\circ} \mathrm{C}$. In total, $140 \mu \mathrm{L}$ of the $10 \%$ suspension was used to extract RNA with the QIAamp ${ }^{\circledR}$ Viral RNA Mini Kit (Qiagen, Venlo, The Netherlands) according to the manufacturer. RNA was used immediately for HEV RT-PCR amplification.

\subsection{HEV RT-PCR and Southern blot hybridization}

For the detection of HEV RNA, a single round RT-PCR with primers ORF2-s1 and ORF2-a1 was used [35]. This RT-PCR yielded a fragment of 197 nucleotides. The RT-PCR fragment was separated in a $2 \%$ agarose gel and HEV RT-PCR fragments were confirmed by Southern blot hybridization [41]. To monitor possible inhibition during RT-PCR, an internal RNA control and a ten-fold serial dilution of undiluted sample RNA were included at the stage of reverse transcription [32]. Three serial tenfold-dilutions of porcine HEV RNA of genotype 3 (GenBank: DQ996399) were used as the positive RT-PCR control. The second serial dilution of control RNA contained a concentration around the detection limit of the RT-PCR. RNase free water was used as the negative control.

\subsection{Transmission model}

Transitions of pigs from the susceptible state $(S)$ to the infectious state $(I)$, and from the infectious state to the recovered state $(R)$ were described by a stochastic SIR-model (e.g. [4, 11]). Pigs were considered susceptible when antibodies against HEV were absent and considered infectious when HEV 
was excreted faecally. Transitions from $S$ to $I$ occur with transmission rate $\beta$, defined as the average number of successful transmissions by an infectious individual per day in a completely susceptible population (where $S /(S+I+R) \approx 1$ ). A modification was made to the SIR-model proposed by Becker [4] by introducing an effect of population size on $\beta$ (frequency dependent transmission model), because this was shown to approximate reality more closely $[9,10]$.

Transitions from $I$ to $R$ in the SIR-model depend on rate of recovery $\alpha$, defined as the reciprocal of the infectious period in days. Since $\beta / \alpha$ gives the estimate for $R_{0}$, rate of transmission and length of the infectious period need to be estimated.

\subsection{Estimation of the rate of transmission}

Use of one-to-one challenges to quantify transmission of a pathogen has the advantage that the occurrence of transmission becomes a binomial parameter (transmission does occur or not).

To estimate $\beta$, each pig was allocated to one of the states $S$ or $I$ at each sampling based on the absence $(S)$ or presence $(I)$ of HEV RNA in faeces. However, due to a delay between actual infection and first HEV-excretion in faeces - the latent period - pigs may already be infected without excreting HEV. Therefore, dpi of first HEV excretion in faeces should be adjusted to the actual moment of infection by subtracting the length of the latent period. Since data on the length of the latent period for HEV in pigs are lacking, we estimated the latent period from data on IV-pigs, where the moment of infection was known. To this end, dpi for the last negative faecal sample and the first positive faecal sample were averaged per IV-pig. The mean of these averages was the estimate for the latent period. If adjustment for the latent period subsequently yielded an estimated moment of infection prior to exposure to an infectious pig, then the moment of infection was set to the first sampling-interval in which contact-exposure occurred.

Since next-generation contact pigs in our experiments sporadically excreted HEV before being contact-exposed to an infectious pig, we needed to consider the transmission between compartments in addition to the transmission within compartments. To estimate both transmission rates, $\beta$ was divided in rate of transmission within compartments $\left(\beta_{w}\right)$ and an additional rate of transmission for transmission between compartments ( $\beta_{a}$ ) (cf. [22]). The rate of transmission between compartments $\left(\beta_{b}\right)$ was the sum of $\beta_{w}$ and $\beta_{a}$. For each susceptible pig, infectious pigs were counted in the own $\left(I_{w}\right)$ and in neighboring $\left(I_{b}\right)$ compartments, with $I_{\text {tot }}$ being $\left(I_{w}+I_{b}\right)$. Furthermore, the fraction of all the infectious pigs that were in the neighboring compartment $\left(I_{b} / I_{t o t}\right)$ was represented by $f$. The probability of infection per unit of time $(p)$ can now be described as a function of $\beta, I, N$, and time interval between samplings $(\Delta t)$ :

$$
p=1-e^{-\left(\beta_{w} \times \beta_{a}^{f}\right) \frac{I_{t o t}}{N} \Delta t}
$$

The value of $p$ is estimated by the expected number of new cases $(\mathrm{E}(C))$ divided by the number of trials in a binomial process (in our situation $S$ ). To obtain linearity in regression parameters, a complementary log-log link function can be applied:

$$
\begin{aligned}
c \log \log \left[\frac{E(C)}{S}\right]= & \log \left(\beta_{w}\right)+f \log \left(\beta_{a}\right) \\
& +\log \left(\frac{I_{\text {tot }}}{N} \Delta t\right)
\end{aligned}
$$

This model was analyzed statistically with STATA $^{\circledR} 9$ (StataCorp LP, College Station, USA) as a generalized linear model with the complementary$\log$-log link function, the term $\log \left(I_{\text {tot }} / N \times \Delta t\right)$ as offset, $f$ as explanatory variable and $S$ as the number of trials in the binomial process.

\subsection{Estimation of the length of the infectious period}

Since $R_{0}$ represents the number of new infections caused by an infected pig during the entire infectious period, the length of the infectious period needs to be estimated. The infectious period was defined as the average number of days that infected pigs excreted HEV, and was estimated with survival analysis [21] from data on contactinfected pigs. Because of the two- or three-day intervals between consecutive samplings, the start of HEV-excretion was calculated as the average of dpi of the last negative and first positive sampling. Analogously, the end of HEV excretion was calculated as the average dpi between last positive and first negative samplings. 


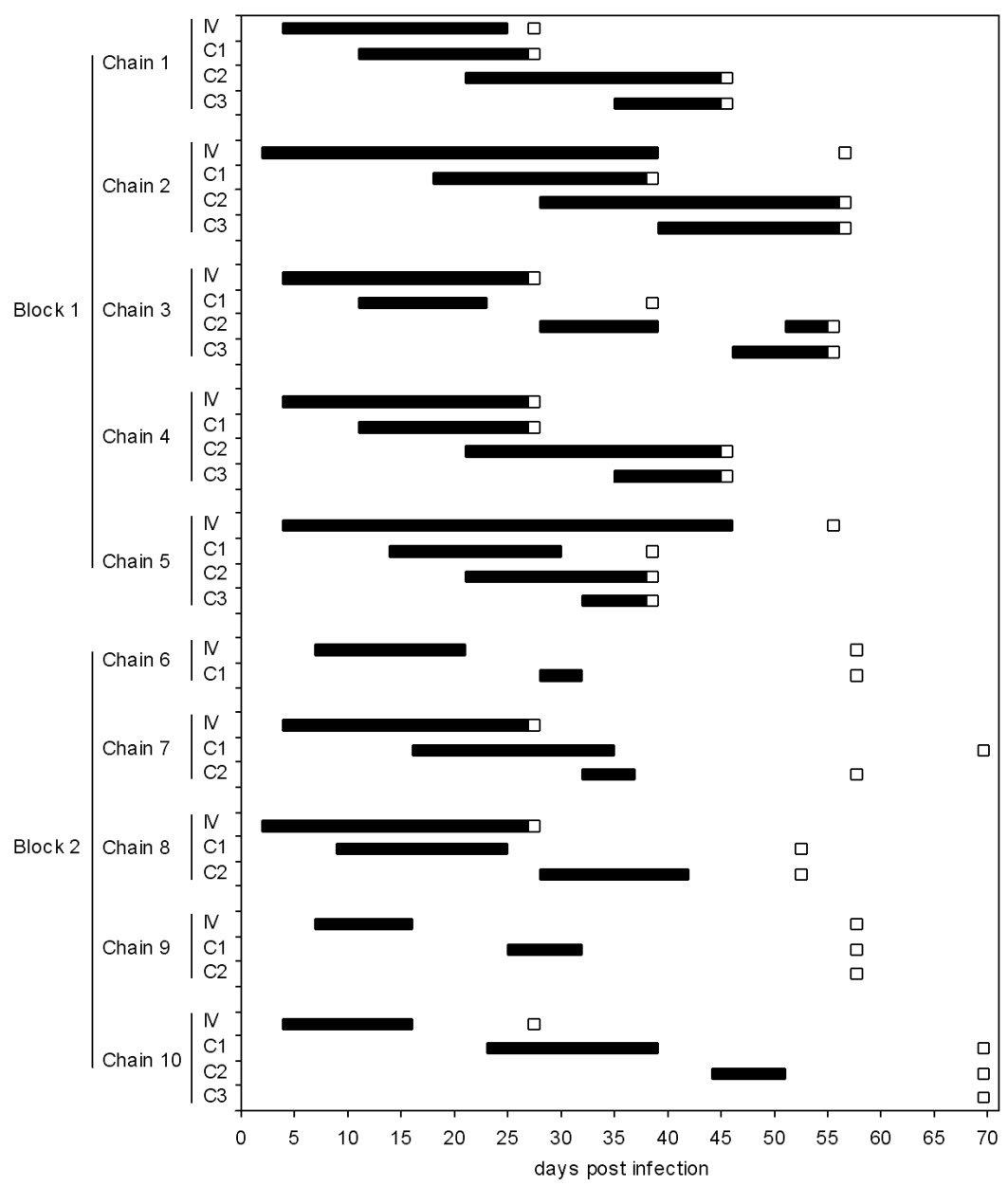

Figure 2. HEV RNA excretion in faeces per pig (IV-C3) for the two blocks. Each set of four, three or two pigs represents a transmission chain. For details on transmission chains, see Figure 1. The euthanization of pigs is represented by ' $\square$ '.

\section{RESULTS}

\subsection{HEV excretion and transmission}

All ten IV-pigs started to excrete HEV in faeces between 0 dpi and 7 dpi (Fig. 2). The average period between HEV inoculation and start of HEV excretion - the latent period - was three days (range: 2-7, median: 3). The latent period did not differ significantly between block 1 and block 2 ( $P_{\text {Kruskal-Wallis }}=$ 0.40). All ten IV-pigs transmitted HEV to C1-pigs (Tab. I). For C1-pigs, periods between exposure to infectious IV-pigs and HEV excretion did not differ significantly between block 1 (9 days) and block 2 (17 days) ( $\left.P_{\text {Kruskal-Wallis }}=0.09\right)$.

The C1-pigs that shed HEV in faeces at three consecutive samplings were transferred to compartments with C2-pigs and HEV excretion was monitored (Fig. 2). HEV transmission occurred to four C2-pigs in block 1 and to three C2-pigs in block 2 . In block 1, one C2-pig excreted HEV before being contact-exposed to a C1-pig. Nevertheless, the 
Table I. Transmission of hepatitis E virus by contact-exposure of susceptible pigs to infectious pigs.

\begin{tabular}{|c|c|c|c|c|c|c|}
\hline & \multicolumn{6}{|c|}{ Transmission from $^{\mathrm{a}}$} \\
\hline & \multicolumn{2}{|c|}{ IV-pigs to C1-pigs } & \multicolumn{2}{|c|}{ C1-pigs to C2-pigs } & \multicolumn{2}{|c|}{ C2-pigs to C3-pigs } \\
\hline & Success & $t^{\mathrm{b}}(\mathrm{SD})$ & Success & $t(\mathrm{SD})$ & Success & $t(\mathrm{SD})$ \\
\hline Block 1 & $5 / 5$ & $9(4)$ & $4 / 4^{c}$ & $3(4)$ & $5 / 5$ & $6(3)$ \\
\hline Block 2 & $5 / 5^{\mathrm{d}}$ & $17(7)$ & $3 / 4$ & $9(4)$ & $0 / 1^{\mathrm{e}}$ & \\
\hline Total & $10 / 10$ & $13(7)$ & $7 / 8$ & $6(4)$ & $5 / 6$ & $6(3)$ \\
\hline
\end{tabular}

a IV: inoculated; C1: first-generation contact pig; C2: second-generation contact pig; C3: third-generation contact pig.

b Average number of days between exposure and estimated moment of infection.

${ }^{c}$ One C2-pig excreted HEV before being contact-exposed to the C1-pig.

${ }^{\mathrm{d}}$ One $\mathrm{C} 1$-pig excreted HEV in faeces at three consecutive samplings, but HEV excretion was ended at day of transfer to the next compartment.

e Two C3-pigs excreted HEV before being contact-exposed to the C2-pig.

transmission chain was continued by contact exposing a C3-pig to this C2-pig. For C2pigs, periods between exposure to infectious C1-pigs and HEV-excretion did not differ significantly between block 1 (three days) and block 2 (nine days) $\left(P_{\text {Kruskal-Wallis }}=0.10\right)$.

The C2-pigs that shed HEV in faeces at three consecutive samplings were transferred to compartments with C3-pigs and HEV excretion was monitored in both pigs. Hepatitis $\mathrm{E}$ virus was transmitted to five C3-pigs in block 1. In block 2, two C3-pigs excreted HEV before being contact-exposed to a C2-pig. The other C3-pig in block 2 did not excrete HEV in faeces. The average period between exposure and first $\mathrm{HEV}$ excretion by the C3-pigs was six days.

\subsection{Quantification of HEV transmission}

The latent period for IV-pigs of three days was used to adjust the moment of becoming infectious to the actual moment of infection. This resulted in overall maximum likelihood estimates for the rate of transmission within compartments $\left(\beta_{w}\right)$ of 0.66 (95\% CI: 0.32 1.35) per day, and for the rate of transmission between compartments $\left(\beta_{b}\right)$ of $0.02(95 \% \mathrm{CI}$ : 0.01-0.04) per day. There was no observed difference in estimates for $\beta_{w}$ and $\beta_{b}$ between the two blocks, as determined from the difference in maximum likelihood between the models with and without "block" as the explanatory variable $\left(P_{\text {chi-square }}=0.21\right)$.
The period of HEV excretion was observed for ten of 23 pigs; for the other 13 the endpoint of HEV excretion in faeces was not determined (i.e. data were censored). Subsequently, the infectious period was estimated at 49 days (95\% CI: 17-141) in block 1 and at 13 days (95\% CI: 11-17) in block 2. The difference between the two blocks was statistically significant $(P<0.01)$.

Since the main objective of the current study was to assess whether or not HEV is transmitted among pigs, we were interested in whether or not " 1 " is excluded from the $95 \%$ confidence interval of $R_{0}$. By using the estimate of 13 days for the infectious period, we tested the hypothesis that HEV transmission does not occur among pigs conservatively. Since $R_{0}$ is the product of $\beta_{w}$ and the length of the infectious period, this mounts to 8.8 (95\% CI: 4.2-18.8). When the infectious period of 49 days was used to estimate $R_{0}$, then $R_{0}$ equalled 32 (95\% CI: 11.2-92).

\section{DISCUSSION}

Experimental HEV-infection in susceptible pigs has been established through intravenous, oral and intrahepatic inoculation $[3,16,20]$ and through contact-exposure to an infectious pig [20, 26]. Intravenous inoculation is used most often, since it has a higher probability of success compared to oral inoculation [19]. It is, however, not the natural route of 
transmission, which is supposedly faecal-oral transmission. In the current study, we used intravenous inoculation to obtain infectious pigs. Subsequently, we used the intravenously inoculated pigs to generate first-, secondand third-generation contact-infected pigs to estimate the $R_{0}$ for transmission of $\mathrm{HEV}$ in pigs. The $R_{0}$ was estimated as 8.8 and was significantly larger than 1 , which indicates that HEV is able to spread when contacts between pigs occur randomly [7]. Therefore, pigs can be a true animal reservoir for HEV. Whether the status of true animal reservoir applies to field conditions (i.e. pig farms) as well, however, remains to be assessed. Field and experimental conditions differ, amongst others, in the contact-structure between pigs. The contact-structure at the farm-level can be defined at different levels: multiple pigs represent a pen, multiple pens represent a unit, and multiple units represent a barn. The frequency and intensity of contact between pigs at different levels of the contact-structure is different than that for pigs within the same level. This will likely lower the $R_{0}$ on pig farms as compared to the $R_{0}$ in the current experiment.

Recently, Satou and Nishiura [34] used cross-sectional serological data from pigs of different ages to estimate the force of infection for HEV. Using that force of infection estimate, $R_{0}$ was estimated at 4.02-5.17. By calculating $R_{0}$ from the estimated force of infection it was assumed that new infections in pigs were solely caused by infectious pigs. Based on our results, showing that $\mathrm{HEV}$ is indeed transmitted among pigs, this is in retrospect probably a correct assumption. The estimates by Satou and Nishiura [34] are about half our estimate of $R_{0}$. This difference may be due to differences in contact structure between pigs on pig farms and the pigs in our experiment. Furthermore, a different definition of $\mathrm{HEV}$ infection, viz based on seroconversion or virus excretion, may also contribute to different $R_{0}$ estimates. The sensitivity and specificity of the assays used to detect either anti-HEV antibodies or HEV genomes will differ. Furthermore, seroconversion is not detected in all pigs that excrete HEV faecally $[19,20]$. In addition to a false-negative test result, this finding may also indicate the absence of antibody development in some pigs. These pigs will not be considered infected when serological data is used as the definition of infection. Therefore, differences in the $R_{0}$-estimates between the current study and the study by Satou and Nishiura [34] are expected.

On three occasions, contact-pigs excreted $\mathrm{HEV}$ in faeces before being contact-exposed to an infectious pig. One pig showed HEV RNA and anti-HEV antibodies also in faeces and serum, respectively, taken two or three days pre-exposure; the other two pigs did not (data not shown). Since anti-HEV antibody can be detected in serum at the earliest two weeks after intravenous inoculation [14], the seroconverted pig was infected likely more than two weeks before taking the pre-exposure samples. The other two pigs were likely in the initial phase of infection, since only HEV RNA in faeces was detected. The design of compartments and the sequence of sampling of pigs were to minimise the risk of $\mathrm{HEV}$ transmission via direct contact between pigs from neighbouring compartments or via animal technicians. However, HEV transmission by these transmission routes cannot be excluded entirely. In addition, another possible route may be cross-over of HEVcontaminated bedding between compartments due to pig-activity. By including the possibility of HEV transmission from neighbouring compartments in the statistical model, we estimated transmission within and between compartments. The transmission between compartments was estimated to be a factor 30 lower compared to transmission within compartments.

For three contact-infected pigs, the adjustment for the latent period would yield an estimated moment of infection within one day before introduction of the infectious pig. We, however, assumed that these pigs were contact-infected after introduction of the infectious pig, as permitted by the $95 \%$ confidence interval for the latent period. However, these pigs may also have been infected prior to contact-exposure. In that case, the rate 
of transmission between compartments would be increased at the expense of the rate of transmission within the compartment. Indeed, when assuming that these three pigs were infected prior to contact-exposure, the $R_{0}$ was estimated to be 7 (95\% CI: 3.2-16.4) with the $95 \%$ confidence interval excluding " 1 ". Therefore, our assumption does not affect the conclusion that HEV can spread among pigs.

Intravenously inoculated pigs in block 2 received a $0.3 \log _{10}$ lower dose of $\mathrm{HEV}$ compared to the inoculated pigs in block 1 . A dose-response relationship is described for $\mathrm{HEV}$ in pigs, with lower peak HEV-titres in faeces and a delay in first HEV-detection due to a three-log lower dose [26]. We observed no delay in first HEV-detection in faeces for inoculated pigs in block 2 compared to inoculated pigs in block 1 , shown by the absence of a significant difference in the latent period between both blocks. This result was expected, since the difference in dose in our study was marginal compared to the three-log lower dose by Meng et al. [26]. In contrast, a shorter average infectious period for pigs from block 2 compared to pigs from block 1 was observed. Whether dose has an effect on duration of HEV-excretion is currently unknown, but we hypothesize that the different infectious periods are more likely the result of experimental variation (e.g. different genetic composition of pigs or a non-regulated relative humidity) than of the marginal different doses.

The period between exposure of susceptible pigs to infectious pigs and the subsequent $\mathrm{HEV}$-excretion differed for C1-pigs (13 days), and $\mathrm{C} 2$ and C3-pigs (6 days). The C1-pigs were exposed to IV-pigs from inoculation onwards. In contrast, the C2-pigs and C3pigs were exposed to infectious pigs that had already excreted HEV at the last three samplings. Concentrations of HEV RNA in faeces for a third positive faecal sample were generally higher than for the first positive faecal sample (data not shown). A higher concentration of $\mathrm{HEV}$ in faeces may increase the probability of HEV-transmission per unit of time compared to a lower concentration, assuming that the amount of faecal excretion is not negatively correlated with $\mathrm{HEV}$ concentration in faeces. A higher probability of HEV-transmission will on average shorten the period between exposure and infection. This shorter period can be explained in binomial terms by an increased probability of success. An increased probability indicates that the average number of successful transmissions per day $(\beta)$ will be higher, which results in a higher estimate for $R_{0}$. In this perspective, our estimate can be deemed conservative.

The current estimate of $R_{0}$ for HEV was based on a single subtype within genotype 3. Possible strain-dependent infectivity of HEV-subtypes may influence the time period between exposure and infection. Different subtypes of genotype 1 strains of $\mathrm{HEV}$, for instance, infected different numbers of HEV-permissive cells in culture, suggesting differences in infectivity between subtypes within one genotype [13]. Different infectivity will have an effect on transmission of a pathogen, since these differences are related directly to differences in probability of infection per HEV particle [39]. Therefore, a different probability of infection per HEV particle will alter the length of the period between exposure and infection and hence the estimate for $\beta$ and $R_{0}$. Since many different subtypes of $\mathrm{HEV}$ are present among pigs and humans in industrialized countries [23], differences in virulence among different subtypes of HEV genotype 3 need to be assessed.

The period between actual infection and faecal HEV-excretion (in this study defined as the latent period) for $\mathrm{HEV}$ in pigs was estimated from data on intravenously inoculated pigs, because the actual moment of infection was known for these pigs. Oral intake of $\mathrm{HEV}$, however, requires $\mathrm{HEV}$ to pass through the stomach and intestine and this may cause a delay in the start of the infectious period compared to intravenous inoculation [18]. However, by using the latent period for intravenously inoculated pigs, $R_{0}$ was estimated conservatively because a longer latent period results in an estimated actual moment of infection closer to the moment of first contact-exposure. This increases the probability of successful HEV-transmission 
per infectious pig per day and thereby $\beta$. Therefore, the conclusion that HEV is able to spread among susceptible pigs using the latent period for IV-pigs is conservative compared to the use of a longer latent period.

In conclusion, the current experimental design proved useful to study HEV transmission by contact-exposure. By doing so, we estimated $R_{0}$ at 8.8 (95\% CI: 4.2-18.8), indicating that $\mathrm{HEV}$ is able to spread among pigs. Hence, pigs can be a true animal reservoir. Next, the reproduction ratio should be estimated for HEV transmission in pig-farms, where there is a different contact structure compared to the experimental conditions.

Acknowledgements. This research was conducted by order and commissioned by the Dutch Food and Consumer safety Authority (VWA) and supported by the Dutch Product Board for Meat and Eggs (PVE).

Marieke van Es and Juliette Ketelaar are greatly acknowledged for their technical assistance and Peter van Rossum and his team for all animal handlings.

\section{REFERENCES}

[1] Anderson R.M., May R.M., Population biology of infectious diseases: part I, Nature (1979) 280:361-367.

[2] Arankalle V.A., Joshi M.V., Kulkarni A.M., Gandhe S.S., Chobe L.P., Rautmare S.S., et al., Prevalence of anti-hepatitis E virus antibodies in different Indian animal species, J. Viral Hepat. (2001) 8:223-227.

[3] Balayan M.S., Usmanov R.K., Zamyatina N.A., Djumalieva D.I., Karas F.R., Brief report: experimental hepatitis E infection in domestic pigs, J. Med. Virol. (1990) 32:58-59.

[4] Becker N.G., Analysis of infectious disease data. Monographs on statistics and applied probability, London, Chapman and Hall, 1989, p. 234.

[5] Bouwknegt M., Engel B., Herremans M.M., Widdowson M.A., Worm H.C., Koopmans M.P., et al., Bayesian estimation of hepatitis $\mathrm{E}$ virus seroprevalence for populations with different exposure levels to swine in The Netherlands, Epidemiol. Infect. (2008) 136:567-576.

[6] Bradley D.W., Krawczynski K., Cook E.H., McCaustland K.A., Humphrey C.D., Spelbring J.E., et al., Enterically transmitted non-A, non-B hepatitis: serial passage of disease in cynomolgus macaques and tamarins and recovery of disease-associated 27- to 34$\mathrm{nm}$ viruslike particles, Proc. Natl. Acad. Sci. USA (1987) 84:6277-6281.

Page 10 of 11 (page number not for citation purpose)
[7] De Jong M.C., Diekmann O., A method to calculate-for computer-simulated infections-the threshold value, $R_{0}$, that predicts whether or not the infection will spread, Prev. Vet. Med. (1992) $12: 269-285$

[8] De Jong M.C., Kimman T.G., Experimental quantification of vaccine-induced reduction in virus transmission, Vaccine (1994) 12:761-766.

[9] De Jong M.C., Mathematical modelling in veterinary epidemiology: why model building is important, Prev. Vet. Med. (1995) 25:183-193.

[10] De Jong M.C., Diekmann O., Heesterbeek H., How does transmission of infection depend on population size?, in: Mollison D. (Ed.), Epidemic Models, Cambridge, Cambridge University Press, 1995.

[11] Diekmann O., Heesterbeek. J.A., Mathematical epidemiology of infectious diseases: model building, analysis and interpretation, Wiley series in mathematical and computational biology, Chichester, John Wiley \& Sons Ltd, 2000, p. 303.

[12] Drobeniuc J., Favorov M.O., Shapiro C.N., Bell B.P., Mast E.E., Dadu A., et al., Hepatitis E virus antibody prevalence among persons who work with swine, J. Infect. Dis. (2001) 184:1594-1597.

[13] Emerson S.U., Arankalle V.A., Purcell R.H., Thermal stability of hepatitis E virus, J. Infect. Dis. (2005) 192:930-933.

[14] Halbur P.G., Kasorndorkbua C., Gilbert C., Guenette D., Potters M.B., Purcell R.H., et al., Comparative pathogenesis of infection of pigs with hepatitis E viruses recovered from a pig and a human, J. Clin. Microbiol. (2001) 39:918-923.

[15] Herremans M., Vennema H., Bakker J., van der Veer B., Duizer E., Benne C.A., et al., Swine-like hepatitis $\mathrm{E}$ viruses are a cause of unexplained hepatitis in the Netherlands, J. Viral Hepat. (2007) 14:140-146.

[16] Huang Y.W., Haqshenas G., Kasorndorkbua C., Halbur P.G., Emerson S.U., Meng X.J., Capped RNA transcripts of full-length cDNA clones of Swine hepatitis $\mathrm{E}$ virus are replication competent when transfected into huh7 cells and infectious when intrahepatically inoculated into pigs, J. Virol. (2005) 79:1552-1558.

[17] Ijaz S., Arnold E., Banks M., Bendall R.P., Cramp M.E., Cunningham R., et al., Non-travel-associated hepatitis E in England and Wales: demographic, clinical, and molecular epidemiological characteristics, J. Infect. Dis. (2005) 192:1166-1172.

[18] Jameel S., Molecular biology and pathogenesis of hepatitis E virus, Expert Rev. Mol. Med. (1999) 1999:1-16. 
[19] Kasorndorkbua C., Halbur P.G., Thomas P.J., Guenette D.K., Toth T.E., Meng X.J., Use of a swine bioassay and a RT-PCR assay to assess the risk of transmission of swine hepatitis E virus in pigs, J. Virol. Methods (2002) 101:71-78.

[20] Kasorndorkbua C., Guenette D.K., Huang F.F., Thomas P.J., Meng X.J., Halbur P.G., Routes of transmission of swine hepatitis $\mathrm{E}$ virus in pigs, J. Clin. Microbiol. (2004) 42:5047-5052.

[21] Kleinbaum D.G., Survival analysis: a selflearning text, Springer, New York, 1996.

[22] Klinkenberg D., de Bree J., Laevens H., de Jong M.C., Within- and between-pen transmission of classical swine fever virus: a new method to estimate the basic reproduction ratio from transmission experiments, Epidemiol. Infect. (2002) 128:293-299.

[23] Lu L., Li C., Hagedorn C.H., Phylogenetic analysis of global hepatitis $\mathrm{E}$ virus sequences: genetic diversity, subtypes and zoonosis, Rev. Med. Virol. (2006) 16:5-36.

[24] May R.M., Anderson R.M., Population biology of infectious diseases: part II, Nature (1979) 280:455461.

[25] Meng X.J., Purcell R.H., Halbur P.G., Lehman J.R., Webb D.M., Tsareva T.S. et al., A novel virus in swine is closely related to the human hepatitis E virus, Proc. Natl. Acad. Sci. USA (1997) 94:9860-9865.

[26] Meng X.J., Halbur P.G., Shapiro M.S., Govindarajan S., Bruna J.D., Mushahwar I.K., et al., Genetic and experimental evidence for cross-species infection by swine hepatitis E virus, J. Virol. (1998) 72:9714 9721.

[27] Meng X.J., Dea S., Engle R.E., Friendship R., Lyoo Y.S., Sirinarumitr T., et al., Prevalence of antibodies to the hepatitis $\mathrm{E}$ virus in pigs from countries where hepatitis $\mathrm{E}$ is common or is rare in the human population, J. Med. Virol. (1999) 59:297-302.

[28] Meng X.J., Wiseman B., Elvinger F., Guenette D.K., Toth T.E., Engle R.E., et al., Prevalence of antibodies to hepatitis $E$ virus in veterinarians working with swine and in normal blood donors in the United States and other countries, J. Clin. Microbiol. (2002) 40:117-122.

[29] Pina S., Buti M., Cotrina M., Piella J., Girones R., HEV identified in serum from humans with acute hepatitis and in sewage of animal origin in Spain, J. Hepatol. (2000) 33:826-833.

[30] Purcell R.H., Emerson S.U., Animal models of hepatitis A and E, ILAR J. (2001) 42:161-177.

[31] Reyes G.R., Purdy M.A., Kim J.P., Luk K.C., Young L.M., Fry K.E., Bradley D.W., Isolation of a cDNA from the virus responsible for enterically transmitted non-A, non-B hepatitis, Science (1990) 247:1335-1339.

[32] Rutjes S.A., Lodder W., Bouwknegt M., De Roda Husman A.M., Increased hepatitis E virus prevalence on Dutch pig farms from 33 to $55 \%$ by using appropriate internal quality controls for RT-PCR, J. Virol. Methods (2007) 143:112-116.

[33] Saad M.D., Hussein H.A., Bashandy M.M., Kamel H.H., Earhart K.C., Fryauff D.J., et al., Hepatitis E virus infection in work horses in Egypt, Infect. Genet. Evol. (2007) 7:368-373.

[34] Satou K., Nishiura H., Transmission dynamics of hepatitis $\mathrm{E}$ among swine: potential impact upon human infection, BMC Vet. Res. (2007) 3:9.

[35] Schlauder G.G., Desai S.M., Zanetti A.R., Tassopoulos N.C., Mushahwar I.K., Novel hepatitis $\mathrm{E}$ virus (HEV) isolates from Europe: evidence for additional genotypes of HEV, J. Med. Virol. (1999) 57:243-251.

[36] Takahashi K., Kitajima N., Abe N., Mishiro S., Complete or near-complete nucleotide sequences of hepatitis $\mathrm{E}$ virus genome recovered from a wild boar, a deer, and four patients who ate the deer, Virology (2004) 330:501-505.

[37] Takahashi M., Nishizawa T., Miyajima H., Gotanda Y., Iita T., Tsuda F., Okamoto H., Swine hepatitis $\mathrm{E}$ virus strains in Japan form four phylogenetic clusters comparable with those of Japanese isolates of human hepatitis E virus, J. Gen. Virol. (2003) $84: 851-862$.

[38] Takahashi M., Nishizawa T., Tanaka T., Tsatsralt Od B., Inoue J., Okamoto H., Correlation between positivity for immunoglobulin $\mathrm{A}$ antibodies and viraemia of swine hepatitis E virus observed among farm pigs in Japan, J. Gen. Virol. (2005) 86:18071813.

[39] Teunis P.F., Havelaar A.H., The Beta Poisson dose-response model is not a single-hit model, Risk Anal. (2000) 20:513-520.

[40] Van den Berg H., Lodder W., Van der Poel W.H., Vennema H., de Roda Husman A.M., Genetic diversity of noroviruses in raw and treated sewage water, Res. Microbiol. (2005) 156:532-540.

[41] Van der Poel W.H., Verschoor F., van der Heide R., Herrera M.I., Vivo A., Kooreman M., de Roda Husman A.M., Hepatitis E virus sequences in swine related to sequences in humans, The Netherlands, Emerging Infect. Dis. (2001) 7:970-976.

[42] Widdowson M.A., Jaspers W.J., Van der Poel W.H., Verschoor F., de Roda Husman A.M., Winter H.L., et al., Cluster of cases of acute hepatitis associated with hepatitis $\mathrm{E}$ virus infection acquired in the Netherlands, Clin. Infect. Dis. (2003) 36:29-33. 